\title{
A circular RNAs dataset landscape reveals potential signatures for the detection and prognosis of early-stage lung adenocarcinoma
}

\author{
Zhiying Chen, Jiahui Wei, Min Li and Yongjuan Zhao* (1)
}

\begin{abstract}
Background: This study aimed to identify potential circular ribonucleic acid (circRNA) signatures involved in the pathogenesis of early-stage lung adenocarcinoma (LAC).

Methods: The circRNA sequencing dataset of early-stage LAC was downloaded from the Gene Expression Omnibus database. First, the differentially expressed circRNAs (DEcircRNAs) between tumour and non-tumour tissues were screened. Then, the corresponding miRNAs and their target genes were predicted. In addition, prognosis-related genes were identified using survival analysis and further used to build a network of competitive endogenous RNAs (ceRNAs; DEcircRNA-miRNA-mRNA). Finally, the functional analysis and drug-gene interaction analysis of mRNAs in the ceRNA network was performed.

Results: A total of 35 DEcircRNAs (30 up-regulated and 5 down-regulated circRNAs) were identified. Moreover, 135 DEcircRNA-miRNA and 674 miRNA-mRNA pairs were predicted. The survival analysis of these target mRNAs revealed that 60 genes were significantly associated with survival outcomes in early-stage LAC. Of these, high levels of PSMA 5 and low levels of NAMPT, CPT 2 and TNFSF11 exhibited favourable prognoses. In addition, the DEcircRNA-miRNA-mRNA network was constructed, containing 5 miRNA-circRNA (hsa_circ_0092283/hsa-miR-762/ hsa-miR-4685-5p; hsa_circ_0070610/hsa-let-7a-2-3p/hsa-miR-3622a-3p; hsa_circ_0062682/hsa-miR-4268) and 60 miRNA-mRNA pairs. Functional analysis of the genes in the ceRNA network showed that they were primarily enriched in the Wnt signalling pathway. Moreover, PSMA 5, NAMPT, CPT 2 and TNFSF11 had strong correlations with different drugs.
\end{abstract}

Conclusion: Three circRNAs (hsa_circ_0062682, hsa_circ_0092283 and hsa_circ_0070610) might be potential novel targets for the diagnosis of early-stage LAC.

Keywords: Lung adenocarcinoma, Circular RNAs, Survival analysis, Functional analysis, Gene-drug interaction analysis

\footnotetext{
* Correspondence: zyj1987@jlu.edu.cn

Respiratory Department, The Third Hospital of Jilin University, No. 126. Xiantai

Street, Changchun 130033, Jilin, China
}

(c) The Author(s). 2021 Open Access This article is licensed under a Creative Commons Attribution 4.0 International License, which permits use, sharing, adaptation, distribution and reproduction in any medium or format, as long as you give appropriate credit to the original author(s) and the source, provide a link to the Creative Commons licence, and indicate if changes were made. The images or other third party material in this article are included in the article's Creative Commons licence, unless indicated otherwise in a credit line to the material. If material is not included in the article's Creative Commons licence and your intended use is not permitted by statutory regulation or exceeds the permitted use, you will need to obtain permission directly from the copyright holder. To view a copy of this licence, visit http://creativecommons.org/licenses/by/4.0/ The Creative Commons Public Domain Dedication waiver (http://creativecommons.org/publicdomain/zero/1.0/) applies to the data made available in this article, unless otherwise stated in a credit line to the data. 


\section{Background}

Lung cancer remains a serious public health problem across the world with a relatively high mortality rate (approximately 1.7 million deaths each year) [1]. Currently, nearly $80 \%$ patients undergoing primary lung cancer have been diagnosed with non-small cell lung cancer (NSCLC). Lung adenocarcinoma (LAC) is a common subtype of non-small cell lung cancer and accounts for around $40 \%$ of all lung cancer cases [2]. Although surgical resection has been the most efficient therapy option for early-stage LAC, the reported 5-year survival rates for patients remains unsatisfactory $(<15 \%)$ [3]. Therefore, there is an urgent need to identify effective diagnostic signatures correlated with the initiation and development of early-stage LAC.

Circular RNAs (circRNAs) are a subclass of non-coding covalent closed circular RNAs generated by alternative splicing [4]. Overwhelming evidence has shown that circRNAs are evolutionarily conserved and function as miRNA sponges or competitive endogenous RNAs (ceRNAs) to regulate gene transcription [5]. In recent decades, an increasing number of studies have suggested that circRNAs may play crucial roles in cancers, such as hepatocellular carcinoma and NSCLC [6, 7]. Furthermore, microarraybased RNA microarray profiling contributes substantially to systematically screen promising biomarkers that are involved in cancer progression [8]. Zhu et al analysed a circRNA microarray dataset and found that 59 circRNAs had differential expression in LAC samples than in nontumour samples. Further, they noted that in most cases, up-regulated hsa_circ_0013958 was strongly related to the TNM stage and lymphatic metastasis, indicating that this circRNA might be a potential target for the early detection of LAC [9]. Chen et al profiled three non-coding RNAs expression datasets of NSCLC and emphasised that hsa circ_0078767/hsa_miR-330-3p/ RASSF1A axis served significant roles in cell proliferation and invasion of NSCLC [10]. A previous study performed a circRNA microarray analysis of early-stage LAC using GSE101684 set and identified 357 differentially expressed circRNAs (DEcircRNAs). Furthermore, the altered expression of circRNA (hsa_circRNA_404833) was validated using real-time quantitative reverse transcription polymerase chain reaction (qRT-PCR) methods and is predicted to interact with miR-149-5p that was associated with LAC development [11]. However, a comprehensive bioinformatics analysis based on this dataset has not been conducted.

Herein, we re-analysed the circRNA microarray dataset (GSE101684) to identify novel diagnostic and prognostic biomarkers for the management of early-stage LAC. The DEcircRNAs were extracted between tumour and non-tumour tissues; thereafter, predictive analyses of miRNAs and their target genes were performed. The survival analysis was performed to identify prognosis-related genes; then, the DEcircRNA-miRNA-mRNA network was constructed. Finally, the functional analysis and drug-gene interaction analysis were performed to screen novel therapeutic targets for LAC treatment. We believe that our findings will provide new insights into the involvement of circRNAs in the pathogenesis of early-stage LAC.

\section{Methods}

\section{Data source and DEcircRNA screening}

The circRNA expression data (GSE101684) of early-stage LAC and the corresponding annotation files were downloaded from the National Centre for Biotechnology Information Gene Expression Omnibus (NCBI-GEO) repository (http://www.ncbi.nlm.nih.gov/geo/). This dataset contained eight samples (four tumour tissues and paired adjacent normal tissues of patients with early-stage LAC) and was generated using the GPL21825 074301 Arraystar Human CircRNA microarray V2 sequencing platform. Then, the raw circRNA expression data were pre-processed using the $\mathrm{R}$ limma package, including background correction, normalisation and concentration prediction [12]. The probes were annotated to the corresponding circRNAs by combing the matrix data with the platform annotation files. If multiple probes mapped to the same circRNA, the average value of these probes was considered as the expression value of the circRNA. Linear model-experience Bayesian statistics using the limma package in $\mathrm{R}$ combined with t-tests were used for nonspecific filtration of the expression profile data, and the DEcircRNAs were determined. The cut-off criteria of the adjusted $P$-value (adj. $P$-value) was set at 0.05 , and the criterion of fold change was set at $\left|\log _{2} \mathrm{FC}\right|>1.5$. The heatmap and volcano plot of the DEcircRNAs were drawn using $R$ pheatmap and ggplot2 package, respectively.

\section{Prediction of miRNAs regulated by DEcircRNAs and miRNA-target genes}

First, the circRNAs and human mature miRNAs sequencing files with FASTA format were obtained on the basis of the circBase and miRBase databases. Then, miRanda (version 3.3a; https://omictools.com/miranda-tool) was used to predict the interactions between miRNAs and circRNAs. The miRNA-DEcircRNA pairs were further extracted with a score value of $>150$ and an energy value of $<-30$ and visualised using Cytoscape. Subsequently, we obtained the top five circRNAs in the miRNA-DEcircRNA regulatory network as per the degree. Furthermore, the top 5_circRNAmiRNA network was constructed, and the miRNAs in this sub-network were screened using a threshold score value of $\geq 170$. Following this, miRWalk 2.0 (http://zmf.umm.uniheidelberg.de/apps/zmf/mirwalk2/) was employed to predict the target genes of extracted miRNAs above based on seven databases (miRWalk, miRanda, miRanda, miRDB, miRMap, Pictar2, RNA22 and Targetscan) [13]. In order to further 
Table 1 Premiers used in QRT-PCR

\begin{tabular}{ll}
\hline Primer & sequences(5'-3') \\
\hline hsa_circ_0062682-hF & TGCCTCACCAAGTGGACAA \\
hsa_circ_0062682-hR & GGGCTTCAGCGACAGGT \\
hsa_circ_0092283-hF & ACGGCAGAGCTGGCCTTGGA \\
hsa_circ_0092283-hR & AGGAAGGTGGCAGCAGGA \\
hsa_circ_0070610-hF & GCTGGACAAAGGATGACG \\
hsa_circ_0070610-hR & GATGGGCTTGGTAGGTGA \\
GAPDH-hF & TGACAACTTTGGTATCGTGAAGG \\
GAPDH-hR & AGGCAGGGATGATGTTCTGGAGAG \\
\hline
\end{tabular}

screen the LAC-related miRNA-mRNA pairs in the obtained miRNA-mRNA pairs, we took the intersection of the targeted genes (mRNA) in the previous step and the LACrelated genes included in the CTD database [14]. Finally, the miRNA/LAC-associated mRNA interactions were determined.

\section{Survival analyses}

The mRNA data and relevant clinical information of early-stage LAC samples were first downloaded from The Cancer Genome Atlas (TCGA) database, and these data were labelled as the TCGA dataset. Then, the expression value and survival information of mRNAs in miRNA/LAC-related mRNA network were extracted from the TCGA dataset. All the mRNAs were split into the high-expression and low-expression groups as per median expression, using the $\mathrm{R}$ survival package (version 2.42-6; https://cran.r-project.org/web/packages/survival/ index.html) [15]. Moreover, the overall survival (OS) was computed using the Kaplan-Meier (KM) survival method to assess the prognostic value of the LAC-related genes. The correlation coefficient $p<0.05$ showed that mRNA was significantly related to prognosis.

\section{Construction of DEcircRNA-miRNA-mRNA network and functional analysis}

In the final step, we obtained the mRNAs that were significantly associated with prognosis. We screened the miRNA-mRNA pairs related to these mRNAs. Then, we integrated these relationship pairs with the previously obtained circRNA-miRNA relationship pairs. Using the Cytoscape, we constructed a IncRNA-miRNA-mRNA network. Further, the Kyoto Encyclopedia of Genes and Genomes (KEGG) enrichment analysis and Gene OntologyBiological Process category (GO-BP) functional annotation analysis of these genes were performed using the $\mathrm{R}$ clusterprofiler package [16-18]. We have only showed the top five GO-BPs and pathways.

\section{Drug-gene interaction analysis}

The Drug-Gene Interaction database (DGIdb) is an online resource and provides gene druggability information

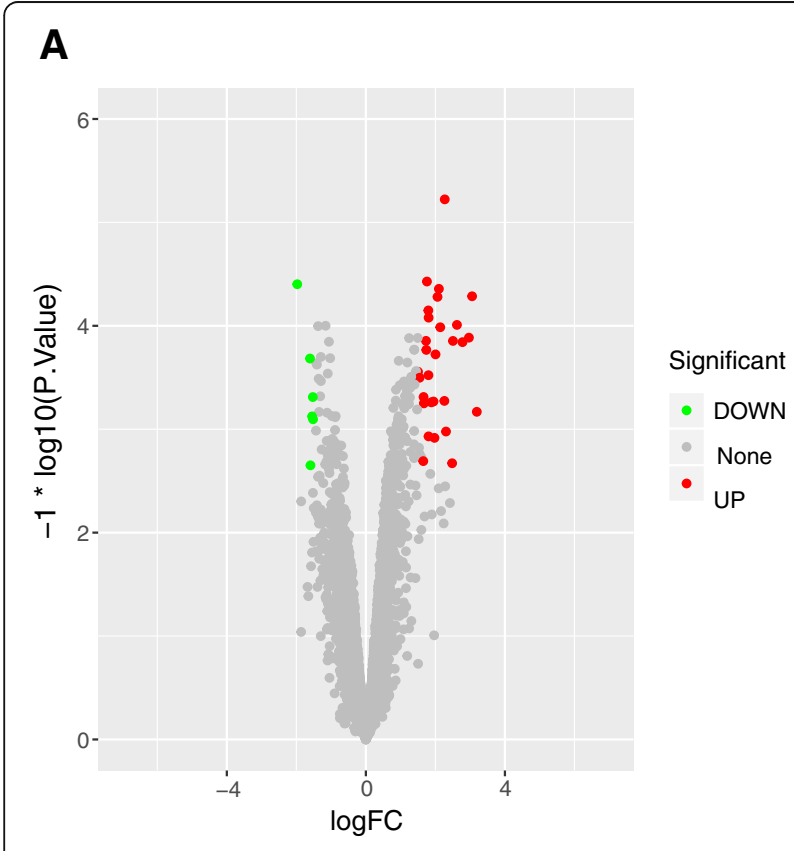

\section{B}

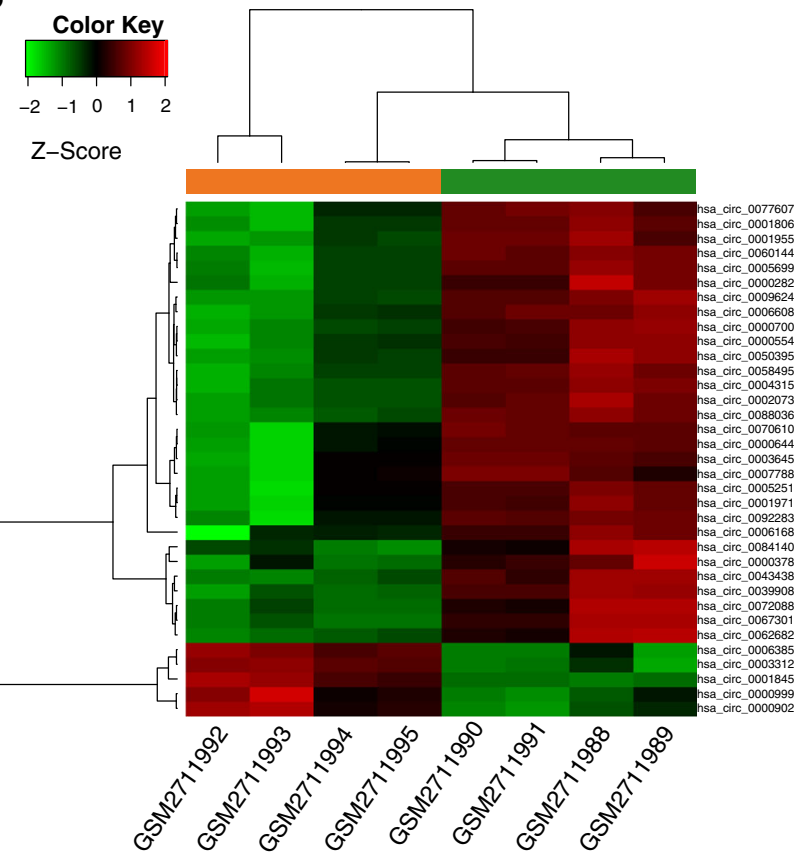

Fig. 1 The differential expression analysis of circRNAs. a The volcano plot of differentially expressed circRNAs. Red and green dots respectively represent the up-regulated circRNAs and down-regulated circRNAs. $\mathbf{b}$ The heatmap of differentially expressed circRNAs 
[19]. We used the DGIdb (version 2.0; http://www.dgidb. $\mathrm{org} /$ ) database to predict the relationships between drugs and genes in the ceRNA network [20]. The FDA and DrugBank were selected as drug databases and the interaction type of NA was excluded from this study. Finally, the drug-gene network was built using Cytoscape.

\section{circRNA detection using $q R T-P C R$}

Total RNA from all the LAC tissues (20) and paired adjacent tissues (20) was extracted using TRIzol Reagent (Invitrogen, Carlsbad, CA, USA). Quantity and quality of RNA were determined spectrophotometrically at 260 and $280 \mathrm{~nm}$, respectively. The integrity and contamination were confirmed using denaturing agarose gel electrophoresis. qRT-PCR methods were performed using an ABI ViiA7 utilising SYBR Premix Ex Taq II (Tli RNaseH Plus) (Takara) as per the manufacturer's instructions. Primers (Table 1) were synthesised by Sangon Biotech (Shanghai, China). The data were analysed using the comparative cycle threshold $(\triangle \mathrm{CT})$ method after three independent experiments. All the results are expressed as the mean \pm standard deviation (SD) values.

\section{Results}

Identification of DEcircRNAs

A total of 35 DEcircRNAs (30 up-regulated DEcircRNAs and 5 down-regulated DEcircRNAs) were identified from the LAC tumour tissues and the adjacent normal tissues as per the screening criteria described in the Methods section. As shown in Fig. 1, the clustering analysis revealed that these DEcircRNAs significantly discriminated LAC and healthy control samples.

\section{Predicting DEcircRNA-miRNA and miRNA-target gene pairs} The miRNAs targeted by DEcircRNAs were first predicted as per the above-mentioned methods. The results indicated that there were 135 DEcircRNAmiRNA pairs among 25 up-regulated DEcircRNAs, 4 down-regulated DEcircRNAs, and 115 miRNAs (Fig. 2). Then, the top five DEcircRNAs in the DEcircRNA-miRNA network were extracted, including up-regulated hsa_circ_0062682, up-regulated hsa_circ_ 0092283, up-regulated hsa_circ_0070610, up-regulated hsa_circ_0005699 and down-regulated hsa_circ 0000902. Then, 772 miRNA-target gene pairs were

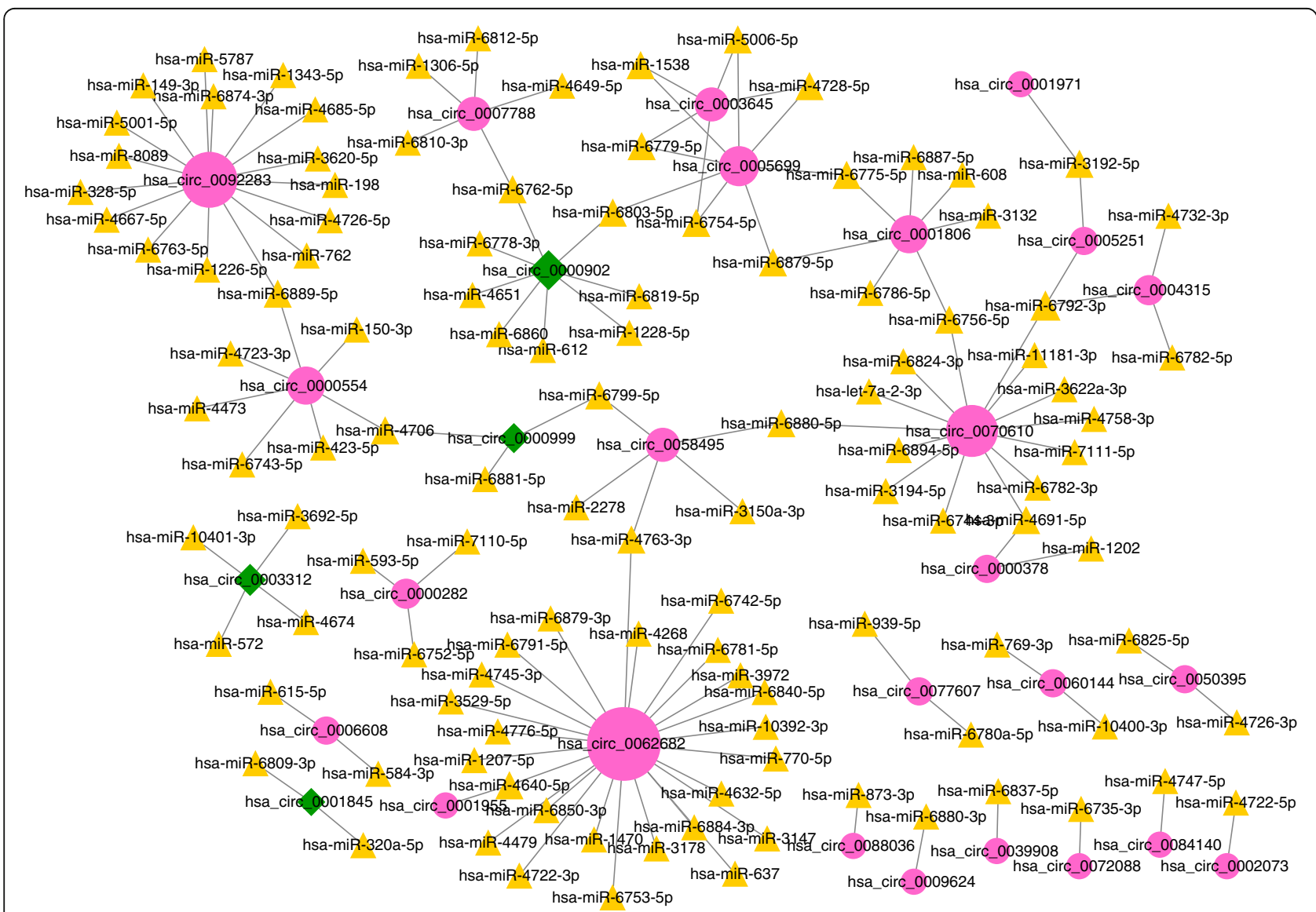

Fig. 2 The differentially expressed circRNA-miRNA regulatory network. Red and green nodes separately represent up-regulated circRNAs and down-regulated circRNAs. The yellow triangles show the predicted miRNAs. A larger node represents a higher degree 
determined using the prediction of miRWalk2.0. Meanwhile, the LAC-related genes were also downloaded from the CTD database. Finally, a total of 674 miRNA-gene interactions were obtained by screening the overlapped genes between target genes in the miRNA-target gene network and the LAC-related genes.

\section{Survival analysis}

To further evaluate the diagnostic values of LAC-related genes in the miRNA-gene network, we downloaded the expression data and clinical information of early-stage LAC patients from the TCGA database. We only included the data of early-stage LAC in the TCGA dataset. A total of 385 samples were used for the final analyses. Thus, the

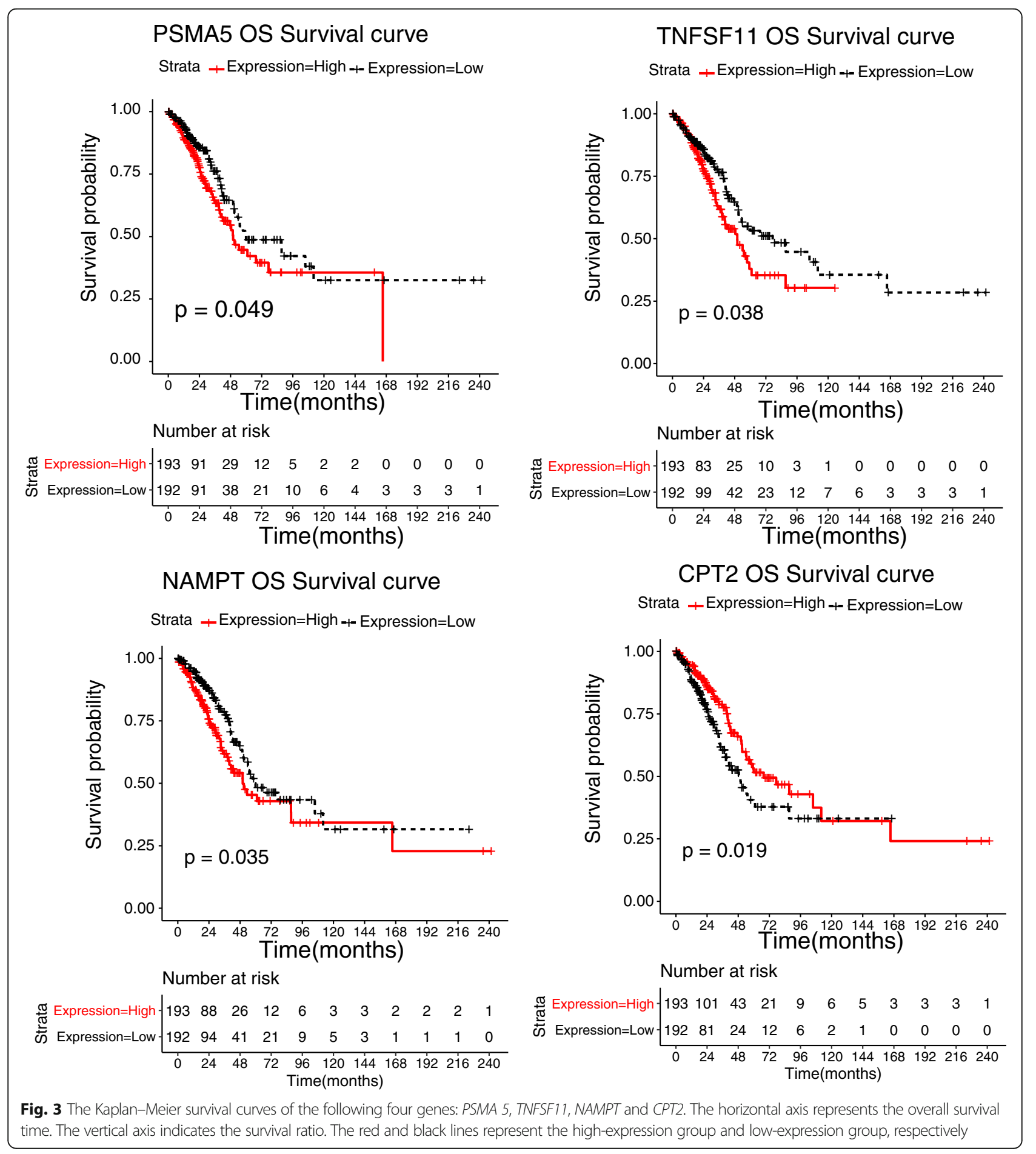


survival analysis of these LAC-related genes was performed; the results showed that 60 genes were closely correlated with the clinical outcomes of LAC patients. The KM survival curves of four genes are shown in Fig. 3, including Proteasome $\alpha 5$ subunit (PSM 5), tumour necrosis factor superfamily member 11 (TNFSF11), Nicotinamide phosphoribosyltransferase (NAMPT) and carnitine palmitoyltransferase 2 (CPT2). We noted that the up- regulated TNFSF11, PSM 5 and NAMPT were strongly associated with worse prognosis. However, higher expression levels of $C P T 2$ exhibited favourable survival outcomes.

CircRNA-miRNA-mRNA network and functional analyses A total of 62 miRNA-mRNA pairs and 5 miRNA-circRNA pairs were used to build the circRNA-miRNAmRNA network that contained 5 miRNAs (hsa-miR-762,

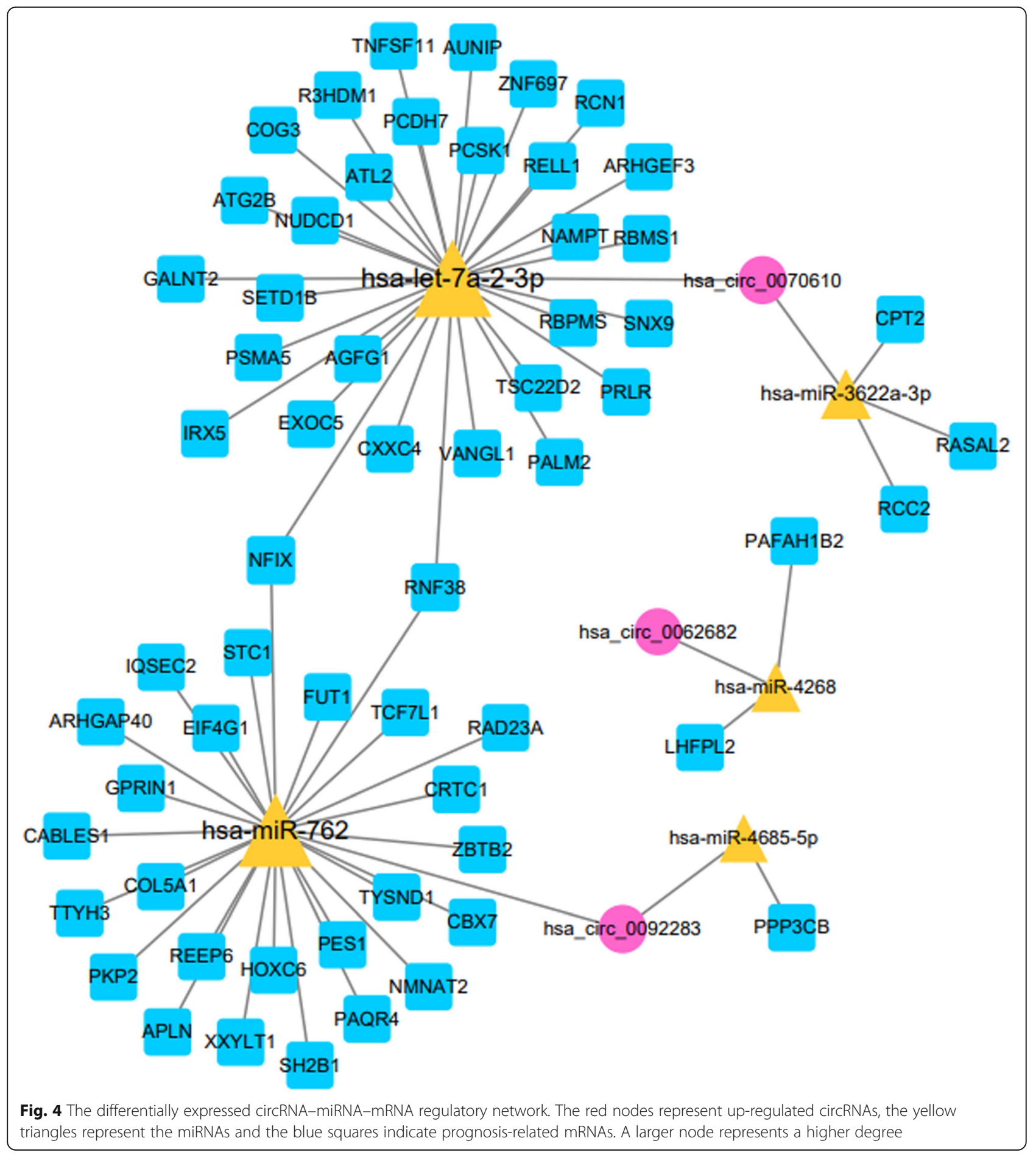




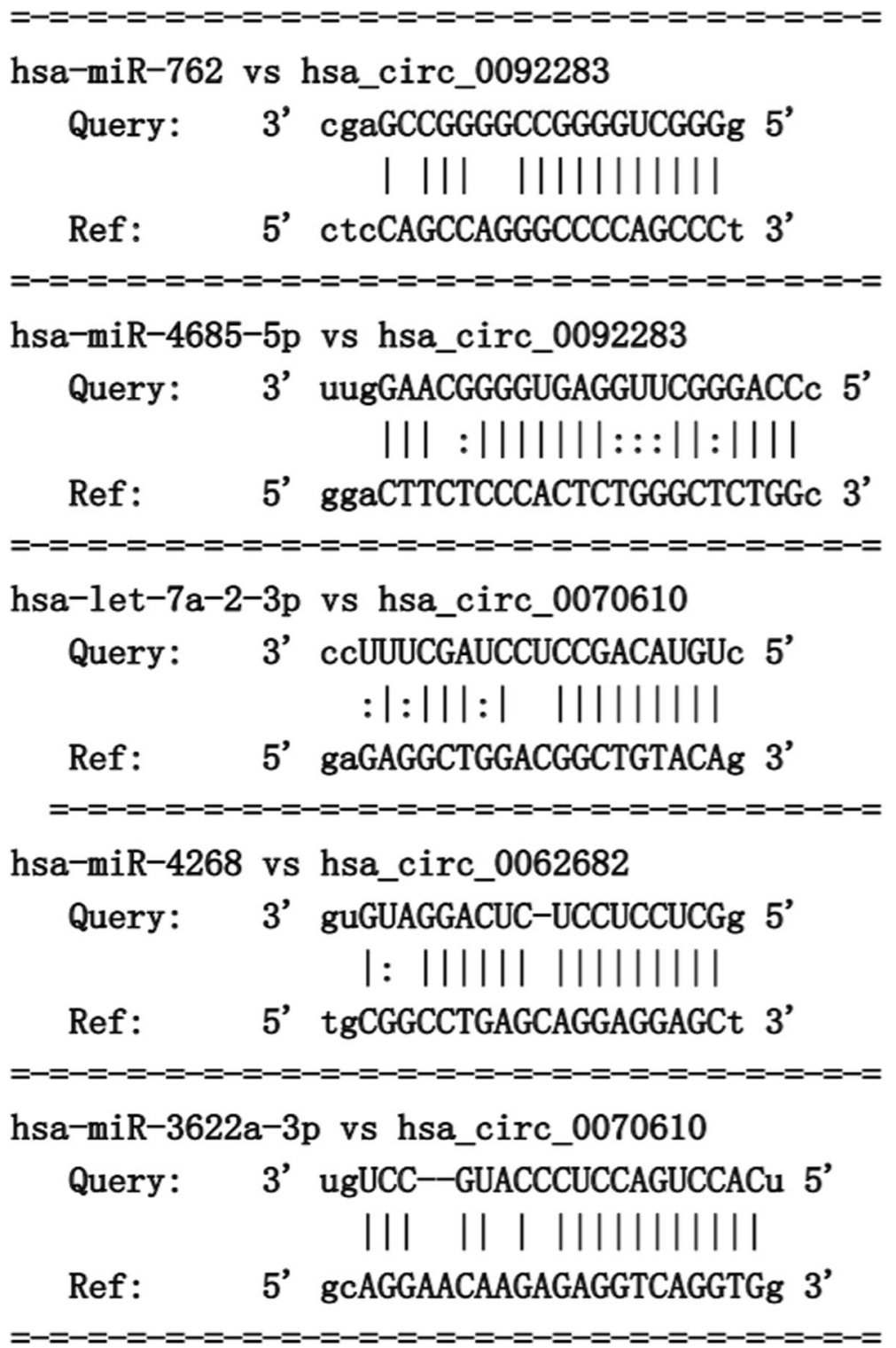

Fig. 5 The map of the binding sites of miRNAs and circRNAs

hsa-miR-4685-5p, hsa-let-7a-2-3p, hsa-miR-4268 and hsa-miR-3622a-3p), 3 up-regulated circRNAs (hsa_circ 0062682, hsa_circ_0092283 and hsa_circ_0070610) and 60 prognosis-related genes (Fig. 4). The prediction analysis of miRNA-binding sites suggested that hsa_circ 0092283 interacted with two miRNAs (hsa-miR-762 and hsa-miR-4685-5p), whereas hsa_circ_0070610 bound strongly to hsa-let-7a-2-3p and hsa-miR-3622a-3p. Meanwhile, hsa_circ_0062682 exhibited a close relationship with has-miR-4268 (Fig. 5). Specific GO categories, such as positive regulation of GTPase activity and protein glycosylation, were significantly enriched in these DE genes (DEGs) (Fig. 6). Moreover, the results of KEGG analysis revealed the potential biological relationship between our gene set and the Wnt signalling pathway (Fig. 6).

\section{Drug-gene interaction prediction}

Our predictive analysis of drug-gene interactions showed that there were 39 drug-gene pairs among 13 genes and 26 drug molecules. As shown in Fig. 7, we can explore the relationship between some genes and drugs.

Furthermore, PSM 5 showed close correlation with the following five drugs: carfilzomib, bortezomib, oprozomib, ixazomib citrate and marizomib. They are all inhibitors. TNFSF11 was associated with lenalidomide, thiocolchicoside and denosumab. Teglarinad chloride was an inhibitor 


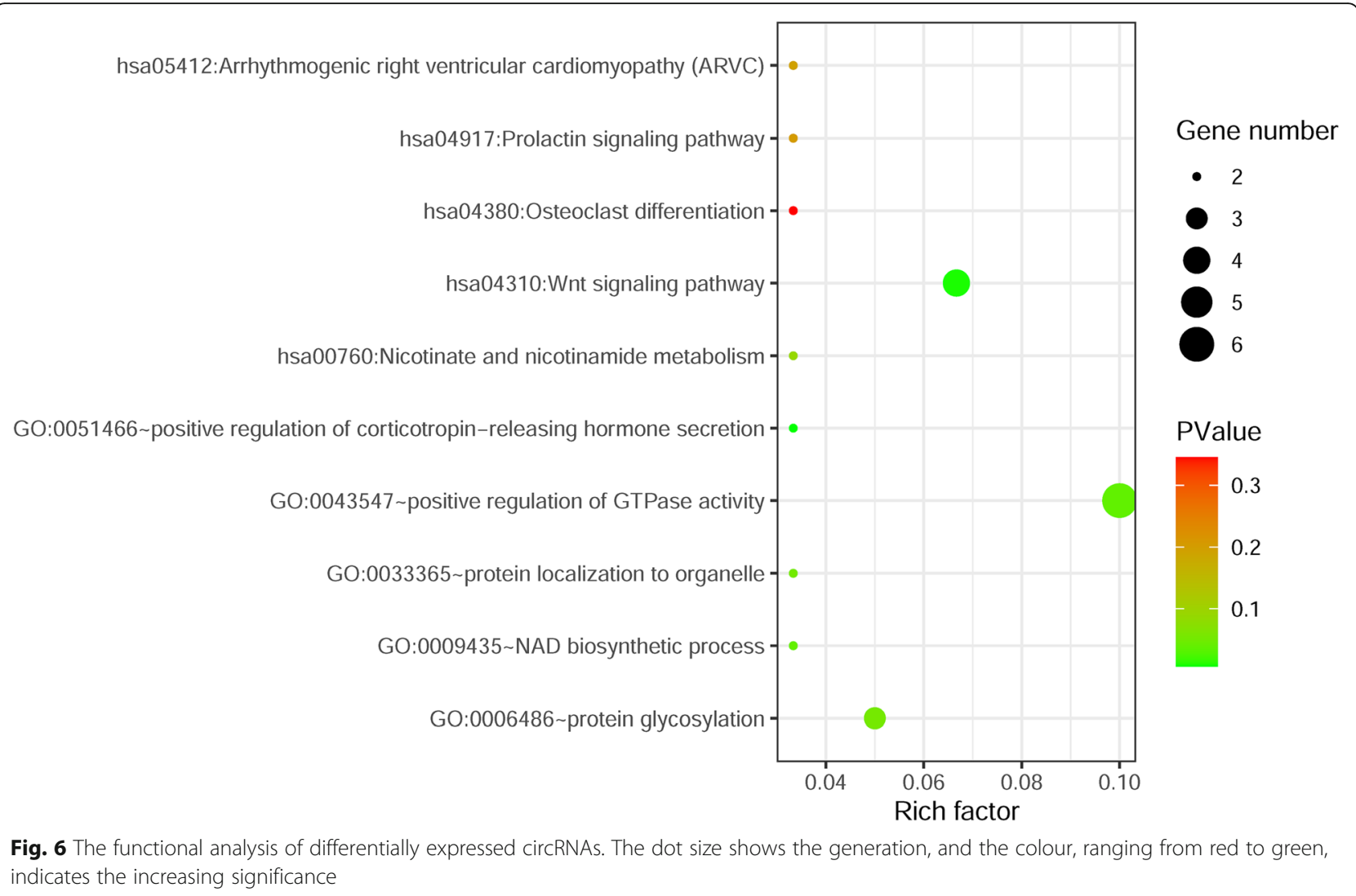

for NAMPT. Moreover, CPT2 closely interacted with perhexiline.

\section{Verification of key circRNAs}

Expression of hsa_circ_0062682 and hsa_circ_0070610 was measured using qRT-PCR in 20 LAC tissues compared with paired adjacent non-tumorous tissues. As shown in Fig. 8, the expressions of hsa_circ_0062682 and hsa_circ_0070610 were significant up-regulated in LAC tissues $(P<0.05)$. However, we did not find any significant difference in the expression of LAC tissues and that of paired adjacent non-tumorous tissues for hsa circ_0092283. In these samples, the expression abundance of hsa_circ_0092283 was very low. We may need to verify this circRNA using more samples.

\section{Discussion}

LAC remains a main cause of cancer-related mortality owing to the high incidence and lack of effective therapy. Thus, it is important to identify potential therapeutic targets for LAC management. Existing evidence has implied that a growing number of tumour-related circRNAs have been found, and these circRNAs displayed abnormal expressions in several types of cancers based on the highthroughput sequencing analyses [21-23]. Moreover, extensive studies have shown that circRNAs functioned as 'miRNA sponges' and that the circRNA-miRNA-mRNA axis might participate in cancer-related pathways [24, 25]. In this study, we performed bioinformatics analysis to systematically screen the molecular markers for LAC diagnosis and treatment using a circRNA expression profile. A total of 35 DEcircRNAs (30 up-regulated DEcircRNAs and 5 down-regulated DEcircRNAs) were identified, and there were three up-regulated DEcircRNAs (hsa_circ 0062682, hsa_circ_0092283 and hsa_circ_0070610) in the circRNA-miRNA-mRNA network.

hsa_circ_0070610 was bound to two target miRNAs (hsa-let-7a-2-3p and hsa-miR-3622a-3p). Research has shown that let-7 members are involved in tumour growth and development. Yanaihara et al previously pointed out that hsa-let-7a-2 expression was dysregulated in lung cancer tissues as compared to that in noncancer tissues; moreover, hsa-let-7a-2 underexpression was related to poor survival [26]. Moreover, NAMPT, TNFSF1 and PSMA 5 were targets of hsa-let-7a-2-3p. These genes also displayed close relationships with multiple drugs, such as teglarinad chloride, denosumab and anastrozole. $\mathrm{Yu}$ et al suggested that TNFSF11 (also known as $R A N K L$ ) expression level was elevated in patients with lung carcinoma and bone metastasis [27]. 

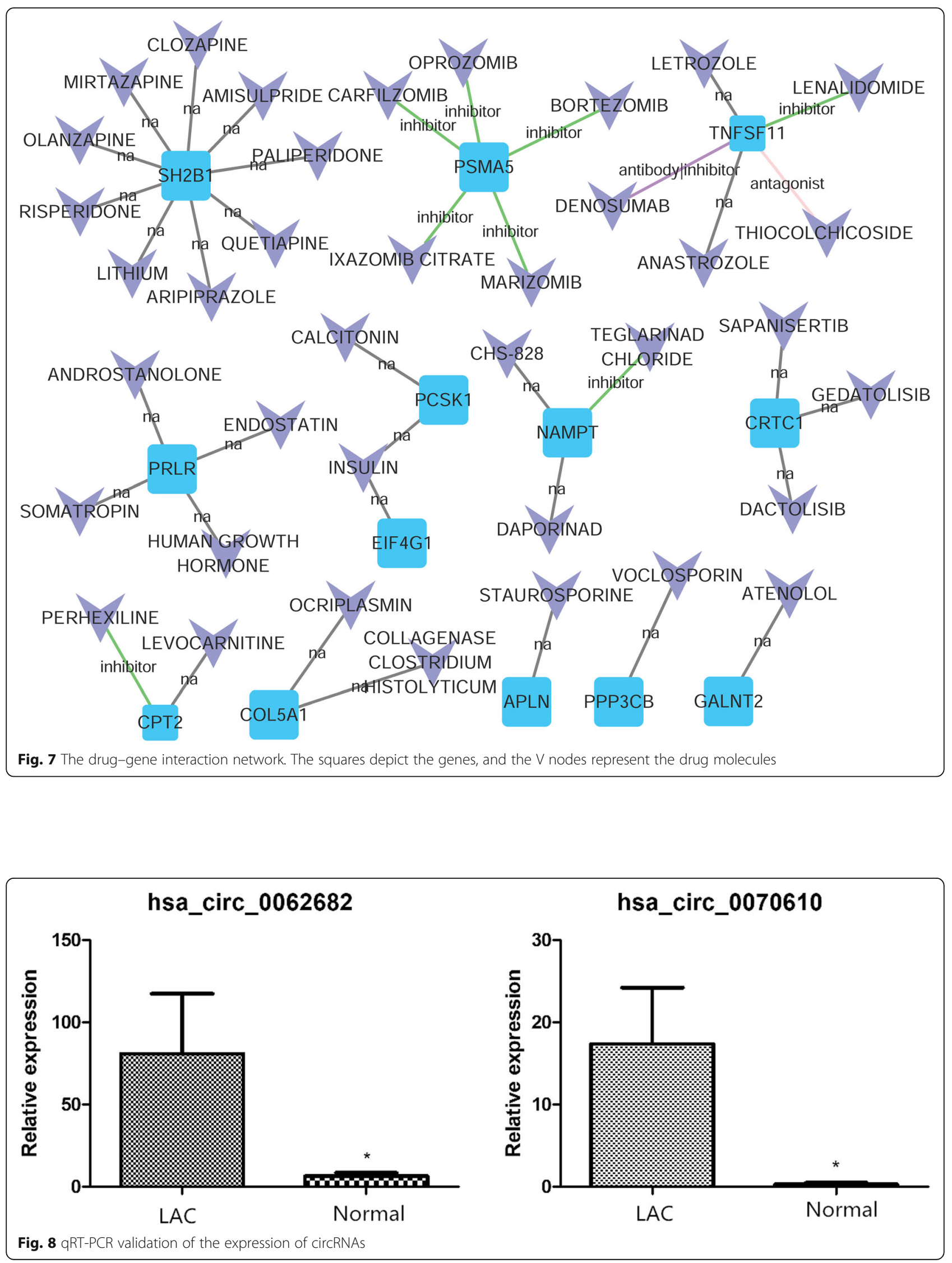
Our survival analysis showed that a lower TNFSF11 level was markedly linked with favourable clinical outcomes in patients with LAC. NAMPT plays an essential role in $\mathrm{NAD}(+)$ biosynthesis in cancer cells and can regulate cellular metabolism, such as altered carbohydrate metabolism in cancer cells [28]. Further, NAMPT is an enzyme essential for NAD+ biosynthesis [28]. A number of NAMPT small molecule inhibitors have been synthesised and applied in clinical practice to date [29]. In our research, teglarinad chloride was found to be an inhibitor for NAMPT. Prostate-specific membrane antigen (PSMA) overexpression is observed in many tumours, such as prostate cancer, gliomas, lung cancer and thyroid cancer [30]. In our research, up-regulated TNFSF11, $P S M 5$ and NAMPT were strongly associated with worse prognosis. However, the involvement of hsa-miR-3622a$3 p$ in the molecular mechanism of LAC has not been studied.

Another circRNA hsa_circ_0092283 exhibited a close association with hsa-miR-4685-5p and hsa-miR-762. Although several trials have shown that hsa-miR-762 were responsible for the occurrence and progression of various cancers, such as bladder cancer and ovarian cancer, whether hsa-miR-4685-5p and hsa-miR-762 participated in the pathogenesis of early-stage LAC remains unknown [31, 32].

In addition, our non-coding RNA network analysis showed that hsa_circ_0062682 closely interacted with hsa-miR-4268. A previous study examined the expression change of miRNAs in patients with metastatic melanoma before and after surgical resection; the results indicated that hsa-miR-4268 had a differential expression [33]. Zhao et al recently showed that hsa-miR-4268 overexpression inhibited the cell proliferation and induced cell apoptosis of gastric cancer cells, suggesting that hsa-miR-4268 might be a tumour suppressor in the development of gastric cancer [34]. However, the direct influences of miR-4268 on LAC progression were being researched at the time of writing this report.

The functional analyses of genes in the circRNAmiRNA-mRNA network revealed that they were predominately correlated with the Wnt signalling pathway and positive regulation of GTPase activity. Activation of the Wnt signalling pathway can promote lung cancer progression and contribute to poor patient prognosis [35]. Many studies have proved that constitutive activation of Wnt/ $\beta$-catenin signalling plays an important role in LAC development [36].

However, this research has certain limitations. First, an integrated bioinformatics analysis needs to be performed on the basis of multiple circRNA microarray datasets to confirm our results. Second, the additional experimental assays were required to validate the results from bioinformatics analyses. Third, the comprehensive clinical information should also be integrated into a large-scale survival analysis. Finally, the potential interactions of non-coding RNAs (circRNA/miRNA/mRNA) need further elaboration in the future.

In sum, three circRNAs (hsa_circ_0062682, hsa_circ_ 0092283 and hsa_circ_0070610) and five miRNA targets (hsa-miR-762, hsa-miR-4685-5p, hsa-let-7a-2-3p, hsa-miR-4268 and hsa-miR-3622a-3p) were probably associated with the development of early-stage LAC. Moreover, PSMA 5, TNFSF11, NAMPT and CPT2 acted as promising diagnostic and prognostic makers for LAC management.

\section{Abbreviations}

NSCLC: Non-small cell lung cancer; circRNAs : Circular RNAs; ceRNAs: Competitive endogenous RNAs; DEcirRNAs: Differentially expressed circRNAs; NCBI-GEO: National Center for Biotechnology Information Gene Expression Omnibus; TCGA: The Cancer Genome Atlas

\section{Acknowledgements}

none.

\section{Authors' contributions}

ZYC and JHW participated in the design of this study, and they both performed the statistical analysis.ML carried out the study and collected important background information. YJZ drafted the manuscript. All authors read and approved the final manuscript.

\section{Funding}

none.

\section{Availability of data and materials}

The expression data were downloaded from the National Centre for Biotechnology Information Gene Expression Omnibus (NCBI-GEO) repository (http://www.ncbi.nlm.nih.gov/geo/). In our research, we used the dataset GSE101684.

\section{Declarations}

Ethics approval and consent to participate

Not applicable.

Consent for publication

Not applicable.

Competing interests

The authors declare no conflict of interest.

Received: 31 May 2020 Accepted: 5 May 2021

Published online: 06 July 2021

\section{References}

1. Bray FFJ, Soerjomataram I, Siegel RL, Torre LA, Jemal A. Global cancer statistics 2018: GLOBOCAN estimates of incidence and mortality worldwide for 36 cancers in 185 countries. CA Cancer J Clin. 2018;68(6):394-424. https://doi.org/10.3322/caac.21492.

2. Ridge CAMA, Ginsberg MS. Epidemiology of lung cancer. Semin Interv Radiol. 2013;30(2):93-8. https://doi.org/10.1055/s-0033-1342949.

3. Youlden DRCS, Baade PD. The international epidemiology of lung cancer: geographical distribution and secular trends. J Thorac Oncol. 2008;3(8):81931. https://doi.org/10.1097/JTO.0b013e31818020eb.

4. Ebbesen KKHT, Kjems J. Insights into circular RNA biology. RNA Biol. 2017; 14(8):1035-45. https://doi.org/10.1080/15476286.2016.1271524.

5. Taborda MIRS, Bernal G. Circular RNAs in colorectal cancer: possible roles in regulation of cancer cells. World J Gastrointest Oncol. 2017;9(2):62-9. https://doi.org/10.4251/wjgo.v9.i2.62. 
6. Wang LLH, Zheng Q, Bo X, Xiao X, Li B. Circular RNA circRHOT1 promotes hepatocellular carcinoma progression by initiation of NR2F6 expression. Mo Cancer. 2019;18(1):119. https://doi.org/10.1186/s12943-019-1046-7.

7. Tan SGQ, Pu W, Guo C, Yang Y, Wu K, Liu Y, et al. Circular RNA F-circEA produced from EML4-ALK fusion gene as a novel liquid biopsy biomarker for non-small cell lung cancer. Cell Res. 2018;28(6):693-5. https://doi.org/1 0.1038/s41422-018-0033-7.

8. Bhattacharjee ARW, Staunton J, Li C, Monti S, Vasa P, Ladd C, et al. Classification of human lung carcinomas by mRNA expression profiling reveals distinct adenocarcinoma subclasses. Proc Natl Acad Sci U S A. 2001; 98(24):13790-5. https://doi.org/10.1073/pnas.191502998.

9. Zhu XWX, Wei S, Chen Y, Chen Y, Fan X, Han S, et al. hsa_circ_0013958: a circular RNA and potential novel biomarker for lung adenocarcinoma. FEBS J. 2017;284(14):2170-82. https://doi.org/10.1111/febs.14132.

10. Chen TYZ, Liu C, Wang L, Yang J, Chen L, Li W. Circ_0078767 suppresses non-small-cell lung cancer by protecting RASSF1A expression via sponging miR-330-3p. Cell Prolif. 2019;52(2):e12548. https://doi.org/10.1111/cpr.12548.

11. Zhao JLL, Wang Q, Han H, Zhan Q, Xu M. CircRNA expression profile in early-stage lung adenocarcinoma patients. Cell Physiol Biochem. 2017;44(6): 2138-46. https://doi.org/10.1159/000485953.

12. Ritchie MEPB, Wu D, Hu Y, Law CW, Shi W, Smyth GK. limma powers differential expression analyses for RNA-sequencing and microarray studies. Nucleic Acids Res. 2015;43(7):e47.

13. Dweep HGN. miRWalk2.0: a comprehensive atlas of microRNA-target interactions. Nat Methods. 2015;12(8):697. https://doi.org/10.1038/nmeth.3485.

14. Davis APGC, Johnson RJ, Sciaky D, McMorran R, Wiegers J, Wiegers TC, et al. The comparative toxicogenomics database: update 2019. Nucleic Acids Res. 2019;47(D1):D948-54. https://doi.org/10.1093/nar/gky868.

15. TM T. Survival analysis [R package survival version $2.41-3]$. Technometrics. 2015:46:111-2

16. Ashburner MBC, Blake JA, Botstein D, Butler H, Cherry JM, Davis AP, et al. Gene ontology: tool for the unification of biology. The gene ontology consortium. Nat Genet. 2000;25(1):25-9. https://doi.org/10.1038/75556.

17. Kanehisa MGS. KEGG: kyoto encyclopedia of genes and genomes. Nucleic Acids Res. 2000;1 (28):27-30.

18. Yu GWL, Han Y, He QY. clusterProfiler: an R package for comparing biological themes among gene clusters. OMICS. 2012;16(5):284-7. https:// doi.org/10.1089/omi.2011.0118

19. Griffith MGO, Coffman AC, Weible JV, McMichael JF, Spies NC, Koval J, et al. DGldb: mining the druggable genome. Nat Methods. 2013;10(12):1209-10. https://doi.org/10.1038/nmeth.2689

20. Wagner AHCA, Ainscough BJ, Spies NC, Skidmore ZL, Campbell KM, Krysiak $K$, et al. DGldb 2.0: mining clinically relevant drug-gene interactions. Nucleic Acids Res. 2016;44(D1):D1036-44. https://doi.org/10.1093/nar/gkv1165.

21. Xu LFX, Hao X, Wang P, Zhang Y, Zheng X, Li L, et al. CirCSETD3 (Hsa_circ_ 0000567) acts as a sponge for microRNA-421 inhibiting hepatocellular carcinoma growth. J Exp Clin Cancer Res. 2019;38(1):98. https://doi.org/10.11 86/s13046-019-1041-2.

22. Li XNWZ, Ye CX, Zhao BC, Li ZL, Yang Y. RNA sequencing reveals the expression profiles of circRNA and indicates that circDDX17 acts as a tumor suppressor in colorectal cancer. J Exp Clin Cancer Res. 2018;37(1):325. https://doi.org/10.1186/s13046-018-1006-X.

23. Hsiao KYLY, Gupta SK, Chang N, Yen L, Sun HS, Tsai SJ. Noncoding effects of circular RNA CCDC66 promote colon cancer growth and metastasis. Cancer Res. 2017;77(9):2339-50. https://doi.org/10.1158/0008-5472.CAN-16-1883.

24. Rong DSH, Li Z, Liu S, Dong C, Fu K, Tang W, et al. An emerging function of circRNA-miRNAs-mRNA axis in human diseases. Oncotarget. 2017:8(42): 73271-81. https://doi.org/10.18632/oncotarget.19154.

25. Wilusz JESP. A circuitous route to noncoding RNA. Science. 2013;340(6131): 440-1. https://doi.org/10.1126/science.1238522.

26. Yanaihara NCN, Bowman E, Seike M, Kumamoto K, Yi M, Stephens RM, et al. Unique microRNA molecular profiles in lung cancer diagnosis and prognosis. Cancer Cell. 2006;9(3):189-98. https://doi.org/10.1016/j.ccr.2006.01.025.

27. Yu MSY, Cui D, Sun Q, Luan B, Zhao D. Chemotherapy effectively suppresses interleukin-20, receptor activator of nuclear factor kappa-B ligand, and osteoprotegerin levels in patients with lung adenocarcinoma and bone metastasis. J Cancer Res Ther. 2016;12(2):963-8. https://doi.org/10.4103/ 0973-1482.179085

28. Tan B, Dong S, Shepard RL, Kays L, Roth KD, Geeganage S, et al. Inhibition of nicotinamide phosphoribosyltransferase (NAMPT), an enzyme essential for NAD+ biosynthesis, leads to altered carbohydrate metabolism in cancer cells. J Biol Chem. 2015;290(25):15812-24. https://doi.org/10.1074/jbc.M114. 632141.

29. Sampath D, Zabka TS, Misner DL, O'Brien T, Dragovich PS. Inhibition of nicotinamide phosphoribosyltransferase (NAMPT) as a therapeutic strategy in cancer. Pharmacol Ther. 2015;151:16-31. https://doi.org/10.1016/j.pha rmthera.2015.02.004

30. Verma P, Malhotra G, Agrawal R, Sonavane S, Meshram V, Asopa RV. Evidence of prostate-specific membrane antigen expression in metastatic differentiated thyroid cancer using 68Ga-PSMA-HBED-CC PET/CT. Clin NuC Med. 2018;43(8):e265-8. https://doi.org/10.1097/rlu.0000000000002161.

31. Hou RYZ, Wang S, Chu D, Liu Q, Liu J, Jiang L. miR-762 can negatively regulate menin in ovarian cancer. Onco Targets Ther. 2017;10:2127-37. https://doi.org/10.2147/OTT.S127872.

32. Lin GSH, Xie H, Zheng $Q$, Shen $Y$, Shi G, Ye D. circLPAR1 is a novel biomarker of prognosis for muscle-invasive bladder cancer with invasion and metastasis by miR-762. Oncol Lett. 2019;17(3):3537-47. https://doi.org/1 0.3892/ol.2019.9970.

33. Latchana NDM, Regan K, Abrams Z, Zhang X, Jacob NK, Gru AA, et al. Alterations in patient plasma microRNA expression profiles following resection of metastatic melanoma. J Surg Oncol. 2018;118(3):501-9. https:// doi.org/10.1002/jso.25163.

34. Zhao LXM, Zhang L, Guo B, Qin Y, Jiang Q, Sun R, et al. MicroRNA-4268 inhibits cell proliferation via AKT/JNK signalling pathways by targeting Rab6B in human gastric cancer. Cancer Gene Ther. 2019;27(6):461-72. https://doi.org/10.1038/s41417-019-0118-6.

35. Guimaraes PPG, Tan M, Tammela T, Wu K, Chung A, Oberli M, et al. Potent in vivo lung cancer Wht signaling inhibition via cyclodextrin-LGK974 inclusion complexes. J Control Release. 2018;290:75-87. https://doi.org/10.1 016/j.jconrel.2018.09.025.

36. Licchesi JD, Westra WH, Hooker CM, Machida EO, Baylin SB, Herman JG. Epigenetic alteration of Wnt pathway antagonists in progressive glandular neoplasia of the lung. Carcinogenesis. 2008;29(5):895-904. https://doi.org/1 0.1093/carcin/bgn017.

\section{Publisher's Note}

Springer Nature remains neutral with regard to jurisdictional claims in published maps and institutional affiliations.

Ready to submit your research? Choose BMC and benefit from:

- fast, convenient online submission

- thorough peer review by experienced researchers in your field

- rapid publication on acceptance

- support for research data, including large and complex data types

- gold Open Access which fosters wider collaboration and increased citations

- maximum visibility for your research: over $100 \mathrm{M}$ website views per year

At $\mathrm{BMC}$, research is always in progress.

Learn more biomedcentral.com/submissions 